\title{
ARTIGOS
}

\author{
Submetido em 14.11.2019. Aprovado em 30.11.2020
}

Avaliado pelo processo double-blind review. Editora Científica Convidada: Rosalia Lavarda

Versão traduzida | DOI: http://dx.doi.org/10.1590/S0034-759020210605x

\section{TRABALHANDO NA INTERSECÇÃO ENTRE AS ABORDAGENS ESTRUTURALISTA E DISCURSIVA: UMA FERRAMENTA METODOLÓGICA PARA ESTUDOS BASEADOS NA PRÁTICA}

\author{
Working at the crossroads of structurationist and discursive approaches: A methodological tool \\ for practice-based studies
Trabajando en la encrucijada de los enfoques estructuracionista y discursivo: Una herramienta metodológica para los estudios basados en la práctica

\author{
Marlei Pozzebon ${ }^{1,2}$ | marlei.pozzebon@hec.ca | ORCID 00oo-0001-5380-908X \\ Natalia Aguilar Delgado1 | natalia.aguilar-delgado@hec.ca | ORCID oooo-0002-2561-1970 \\ Charo Rodriguez³ | charo.rodriguez@mcgill.ca | ORCID oooo-0003-3799-8399 \\ ${ }^{1}$ HEC Montreal, International Business Department, Montreal, Quebec, Canadá \\ ²Fundação Getulio Vargas, Escola de Administração de Empresas de São Paulo, São Paulo, Brasil \\ 3McGill University, Family Medicine Department, Montreal, Quebec, Canadá
}

\section{RESUMO}

Neste artigo, propomos uma ferramenta metodológica que busca contribuir para investigações empíricas da perspectiva da prática em estudos organizacionais. Existe a necessidade de inovar e aprimorar táticas analíticas para teorizar a prática, particularmente para ajudar a conectar interações sociais localizadas em contextos mais amplos. Este artigo propõe o "temporal bracketing” de discursos e inclui um exemplo detalhado que ilustra empiricamente a sua aplicação.

PALAVRAS-CHAVE | Perspectiva da prática, análise crítica do discurso, teoria da estruturação, temporal bracketing, textos em ação.

\section{ABSTRACT}

In this paper we propose a methodological tool that seeks to contribute to the empirical study of practices in organization studies. There is a need for innovating and improving analytical tactics for theorizing about practice, particularly for helping connect localized social interactions to broader contexts. We propose the "temporal bracketing of discourses" tool and provide a detailed example that empirically illustrates its application.

KEYWORDS I Practice-based studies, critical discourse analysis, structuration theory, temporal bracketing, texts-in-action.

\section{RESUMEN}

En este artículo proponemos una herramienta metodológica que busca hacer una contribución al estudio empírico de prácticas en estudios organizacionales. Existe la necesidad de innovar y mejorar las tácticas analíticas para teorizar la práctica, en particular para ayudar a conectar las interacciones sociales localizadas con contextos más amplios. Proponemos la estrategia analítica temporal bracketing para los discursos e incluimos un ejemplo detallado que ilustra empíricamente su aplicación.

PALABRAS CLAVE I Perspectiva de la práctica, análisis crítico del discurso, teoría de estructuración, temporal bracketing, textos en acción. 


\section{INTRODUÇÃO}

A virada da prática tem recebido bastante atenção em estudos organizacionais e na literatura de administração ao longo da última década, particularmente em campos como estratégia (Jarzabkowski, 2008; Lavarda, Canet-Giner, \& Peris-Bonet, 2010; Whittington, 2006), gestão do conhecimento (Orlikowski, 2000) e sistemas da informação (Novak, Brooks, Gadd, Anders, \& Lorenzi, 2012), para citar alguns. As práticas, nesta tradição, referem-se a "rotinas compartilhadas de comportamento, incluindo tradições, normas e procedimentos para pensar, agir e utilizar 'as coisas'” (Whittington, 2006, p. 619), sendo recriadas naquilo que as pessoas fazem e dizem em interações situadas. Seu foco na vida social enquanto produção contínua que emerge por meio das ações recorrentes das pessoas tem trazido à luz uma mudança muito necessária em direção a abordagens agênticas de nosso campo (Feldman \& Orlikowski, 2011). O número de artigos, edições especiais e livros publicados que defendem teorias da prática tem crescido nos últimos anos, servindo para consolidar a posição e a contribuição - tanto conceituais quanto empíricas - dos estudos baseados na prática como uma perspectiva de pesquisa que emerge com força.

Conduzir estudos baseados na prática, no entanto, não é algo livre de dificuldades, justamente devido aos desafios epistemológicos e metodológicos que eles trazem. As teorias da prática constituem uma ampla e complexa paisagem intelectual (Feldman \& Orlikowski, 2011). Em termos de bases teóricas, os pesquisadores orientados para a prática se valem de uma série de teóricos sociológicos, incluindo Giddens (1984), Bourdieu (1990), Foucault (1980) e Schatzki (1996). Tendo “contribuído para o questionamento das abordagens sistemática e determinista que dominaram na sociologia americana até o final dos anos 1970" (Denis, Langley, \& Rouleau, 2007, p. 196), e propondo alternativas para superar os pensamentos dicotômicos clássicos, tais como agência-estrutura, sentido-causa, relativismo-objetivismo, atomismo-holismo, autonomia-tradição e micro-macro (Pozzebon, 2004), as posições teóricas propostas por esses teóricos são frequentemente complexas e difíceis de mobilizar empiricamente (Feldman \& Orlikowski, 2011). As teorias organizacionais focadas em entidades fixas e diagramas estáticos envolvem ferramentas mais instrumentais e menos ambíguas do que as teorias sociais não dicotômicas, que acolhem entidades emergentes e nunca fixas em relações contínuas, dinâmicas e sempre provisórias. Outro grande desafio da teorização da prática reside na conexão entre o "aqui e agora" das interações situadas e o "noutro lugar e então" de contextos mais amplos (Nicolini, 2009). Simplificando, os pontos de vista teóricos emprestados por teóricos da prática são difíceis de aplicar empiricamente.

Duas lentes teóricas parecem ter tido uma grande influência sobre o desenvolvimento da virada da prática: a teoria estruturalista (Jarzabkowski, Balogun, \& Seidl, 2007; Whittington, 2006) e a escola organizacional discursiva, especialmente a análise crítica do discurso (Hardy, 2001; Phillips \& Oswick, 2012). A teoria estruturalista tem se tornado tão proeminente nos estudos organizacionais pelo fato de tentar superar a dualidade estrutura-agência, e por levar o tempo e o espaço a sério, embora pesquisadores desta perspectiva frequentemente prestem mais atenção em atividades individuais e situadas, e menos no contexto político em que essas ações ocorrem (Seidl \& Whittington, 2014; Whittington, 2010). A análise crítica do discurso, considerada como uma das abordagens "sensíveis ao contexto" (Grant, Hardy, Oswick, \& Putnam, 2004), é útil para explicar a conexão entre textos (micro) e contextos políticos amplos (macro). Já foi apontado, no entanto, que o tempo é, frequentemente, uma dimensão negligenciada nesses estudos (Heracleous, 2013; Leitch \& Palmer, 2010).

Em nosso estudo, buscamos oferecer uma ferramenta alternativa para o trabalho empírico sobre estudos baseados na prática ao enriquecermos a intersecção entre a teoria estruturalista e a análise crítica do discurso. Este artigo realiza duas contribuições principais. Primeiramente, damos um passo além no esforço de combi- 
nar a teoria estruturalista e a análise crítica do discurso como um rico caminho teórico e metodológico para os estudos baseados na prática, ao buscarmos entender as interações cotidianas sem perder sua conexão com as influências em nível macro, isto é, políticas, culturais, sociais e outras. Respondemos aos chamados por melhor compreensão das ligações entre os discursos organizacionais, as ações dos agentes e os resultados das mudanças ao longo do tempo na medida em que nos concentramos não apenas nos pontos de convergência entre as teorias (por ex., Heracleous, 2013; Heracleous \& Barrett, 2001), mas, principalmente, na complementaridade potencial dessas abordagens em relação à sua mobilização em trabalhos empíricos. Em segundo lugar, apresentamos uma ferramenta metodológica de passo-a-passo, por meio da qual a fertilização cruzada entre essas tradições pode ocorrer. Descrevemos e ilustramos o temporal bracketing de discursos como uma técnica metodológica que visa ajudar os pesquisadores a estabelecer uma conexão entre interações localizadas e contextos mais amplos, não apenas em aproximação e recuo ("zooming in and out") - para mobilizar a metáfora de Nicolini (2009) - mas também em uma visão ao longo do tempo (zooming over time).

\section{REVISÃO DA LITERATURA}

\section{A teoria estruturalista e seus “dispositivos sensibilizadores” para a análise das práticas sociais}

Desde os anos 1980, a teoria estruturalista tem sido vista como um caminho promissor para a resolução de um longo debate acerca das relações entre estrutura e agência, um desafio não limitado à teoria organizacional, mas relacionado a toda teoria social (Cohen, 1989). Não é nosso objetivo, nesta seção, propor uma visão geral completa da obra de Giddens, uma vez que uma série de escritos abrangentes e autoritativos sobre o tema estão disponíveis (e.g., Cohen, 1989; Held \& Thompson, 1989). Concentramo-nos em revisitar o caminho metodológico proposto pela teoria estruturalista para compreender práticas organizacionais e sociais promissoras, a fim de melhorar o trabalho empírico sobre a virada da prática.

Trabalhos anteriores examinaram como a teoria estruturalista tem sido empiricamente empregada no contexto das pesquisas sobre tecnologia da informação (Pozzebon e Pinsonneault, 2005), e do discurso organizacional (Heracleous, 2013). Seguindo a aplicação de Giddens da teoria estruturalista, essas obras sugerem utilizar dispositivos sensibilizadores de uma maneira seletiva no trabalho empírico, e não como diretrizes detalhadas de procedimentos de pesquisa (Giddens, 1989, p. 294). Na mesma linha, partimos de dois conceitos-chave da teoria estruturalista que utilizamos como dispositivos sensibilizadores: dualidade da estrutura e tempo/espaço.

O conceito de dualidade da estrutura está no cerne da teoria estruturalista, e surge como um dispositivo importante, tanto para o planejamento de uma investigação empírica quando para a interpretação de seus resultados. Giddens parte da conceitualização da estrutura como tendo alguma forma dada ou visível. Embora a estrutura seja o que dá formato à vida social, ela mesma não é esse formato. A estrutura não é sempre tangível, mas existe como traços de memória em nossas mentes, e está em constante recriação pelas ações, reações, vozes e aceitações silenciosas humanas. De maneira semelhante, o autor parte da ideia de agência como algo "contido" dentro do indivíduo. Ele não se refere às intenções das pessoas ao fazer as coisas, mas mais ao fluxo ou padrão de ações das pessoas (Giddens \& Pierson, 1998), que podem ou seguir um dado percurso ou traçar um caminho diferente. Tomadas em conjunto, essas duas noções nos ajudam a compreender o conceito seminal 
de "dualidade da estrutura" (excessivamente apontado, mas frequentemente mal compreendido) que oferece um entendimento mais rico dos encontros sociais. A estrutura está inserida na prática, ou em uma série de práticas, nas quais está recursivamente implicada (Giddens, 1976, 1984).

Além da dualidade agência-estrutura, a noção de tempo/espaço também é central à teoria estruturalista, e é uma característica fundamental na compreensão das propriedades dos sistemas sociais: como as pessoas conceitualizam o tempo e o espaço, e como elas conseguem se organizar no tempo e no espaço (Giddens \& Pierson, 1998). Giddens (1998) destaca a importância de estudar as contextualidades dos padrões institucionalizados de interações no tempo e no espaço, vistas como inerentes à investigação da reprodução social. Argumenta que toda pesquisa social possui necessariamente dimensões culturais, etnográficas ou antropológicas, que, no entanto, são frequentemente negligenciadas nos estudos sociais. Entretanto, esses elementos-chave da teoria estruturalista permanecem subutilizados, mais especificamente a interpenetração da temporalidade relativa às práticas, indivíduos e instituições (Heracleous, 2013).

Exemplos recentes do uso dos conceitos da teoria estruturalista em estudos baseados na prática podem ser encontrados na linha de pesquisa da estratégia-como-prática (Jarzabkowski, 2008), na qual a influência das ideias de Guiddens pode ser reconhecida em trabalhos seminais referenciados na área (Seidl \& Whittington, 2014). Jarzabkowski (2008) sugere que a ligação entre os níveis micro (atividades de strategizing) e macro (a sociedade mais ampla) é mais bem compreendida quando nos concentramos nas "práticas-em-uso da gestão como sendo a unidade básica de análise" (Jarzabkowski, 2004, p. 551). Entretanto, estudiosos da estratégia-como-prática, inspirados ou não no estruturalismo, tendem a negligenciar o contexto estrutural social (Whittington, 2010), levando a relatos acríticos e apolíticos (Carter, Clegg \& Korngerger, 2008).

De maneira semelhante, Seidl e Whittington (2014) apontam a necessidade de mais pesquisas da prática que efetivamente conectem atividades "locais" de strategizing a fenômenos sociais "maiores", em um esforço para superar a tendência ao "microisolacionismo", pelo qual um caso empírico local é "interpretado inteiramente em termos do que está evidentemente presente, desvinculado dos fenômenos maiores que o possibilitam" ( $p$. 1408). Surge uma oportunidade para pesquisadores da prática ampliarem seu entendimento das estruturas sociais maiores, porque a perspectiva da prática proporciona uma conexão entre os níveis micro e macro de análise

\section{A análise crítica do discurso e seu potencial para conectar textos-em-ação situados e contextos mais amplos}

Dentro da escola discursiva ampla, temos testemunhado um aumento persistente e crescente na atenção dada à análise crítica do discurso (Lacerda \& Brulon, 2013). A análise crítica do discurso possui uma longa história na sociolinguística (Titscher, Meyer, Wodak \& Vetter, 2000), e passou a exercer influência nos estudos organizacionais ao longo das três últimas décadas (Grant, Keenoy \& Oswick, 2001). Em comum com outras abordagens discursivas que consideram a linguagem não apenas como ferramenta de descrição e comunicação, mas basicamente como um meio de construção da realidade social (Kuhn \& Putnam, 2014), a análise do discurso se caracteriza, por um lado, por sua ênfase nas relações de poder, e, por outro, por sua sensibilidade ao contexto (Grant et al., 2004; Phillips \& Hardy, 2002).

Intertextual por natureza, a análise crítica do discurso permite a identificação de ideias-chave inseridas nas estruturas interpretativas das pessoas, e explica como essas ideias vão moldar e influenciar suas ações e decisões. Ela ajuda a lançar luz sobre a natureza das relações de poder e sua influência sobre os processos 
organizacionais, e é particularmente eficaz para destacar múltiplas vozes e perspectivas, ajudando, assim, a desmistificar pressupostos tomados como certos, e revelando suas consequências em termos de desigualdades de poder prolongadas (Grant et al., 2001, 2004; Phillips \& Hardy, 2002). A análise crítica do discurso é poderosa em entretecer múltiplos níveis de análise em que o discurso é entendido por sua inserção nas práticas socioculturais (Chouliaraki \& Fairclough, 2010; Fairclough, 1992, 1995; Fairclough \& Wodak, 1997).

Fairclough vê a análise crítica do discurso como um "modo de questionamento crítico em que a teoria e a metodologia estão inerentemente ligadas uma à outra" (Chouliaraki \& Fairclough, 2010, p. 1215). 0 quadro conceitual tridimensional de Fairclough examina como o discurso é constitutivo do mundo social por meio de sua análise em três níveis: o exame da linguagem em uso (a dimensão do texto); a identificação dos processos de produção e consumo textuais (a dimensão da prática discursiva); e a consideração dos fatores institucionais em torno do evento e como eles moldam o discurso (a dimensão prática) (Thompson, 2004). Esses três níveis de análise são explicados em mais detalhes na seção em que apresentamos nossa abordagem metodológica.

Embora a análise crítica do discurso busque estabelecer uma conexão entre as atividades microdiscursivas e contextos mais amplos, a maior crítica a esse corpo de literatura é que ele retira a ênfase do papel do tempo (Heracleous, 2013; Leitch \& Palmer, 2010). É por isso que afirmamos ser mais útil combinar os dispositivos sensibilizadores empíricos dos repertórios teóricos estruturalista e discursivo do que mobilizá-los separadamente. Mostramos isso em mais detalhes na próxima seção.

\section{Conectando a teoria estruturalista e a análise crítica do discurso}

Na maior parte do tempo, a teoria estruturalista e a análise crítica do discurso têm sido aplicadas separadamente (Balogun, Jarzabkowski \& Vaara; Jarzabkowski, 2008), mas alguns autores vem tentando combiná-las. Embora esses dois pontos de vista defendam ontologias diferentes, eles ocupam o que autores como Gioia e Pitre (1990) chamam de 'zonas de transição', isto é, zonas em que cada corrente teórica pode aproveitar facetas diferentes mas complementares dos fenômenos organizacionais, criando um diálogo. Duas ilustrações exemplares desse esforço são a Escola de Montreal de comunicação organizacional (Spee \& Jarzabkowski, 2011; Taylor, Cooren, Giroux \& Robichaud, 1996) e a obra de Heracleous e colegas sobre discurso organizacional (Heracleous, 2013; Heracleous \& Barret, 2001).

Reconhecemos de aplicações anteriores pelo menos dois grandes pontos de convergência e uma importante zona de complementaridade. Primeiro, ambas envolvem processos lógicos robustos para compreender os fenômenos sociais. A estrutura está inserida na prática, ou em uma série de práxis, em que é recursivamente implicada ao longo do tempo e em espaços historicamente situados (Giddens, 1984). Embora as ações das pessoas sejam centrais para a maioria das análises estruturalistas, o conceito de discurso possui grande ressonância na obra de Giddens, onde a linguagem é vista não apenas como uma ferramenta funcional, mas como constitutiva da vida social, desempenhando o papel de meio de interações sociais (Heracleous, 2013). Segundo, tanto a abordagem estruturalista quanto a discursiva entendem que o contexto é central para a compreensão das práticas sociais. Giddens (1984) argumenta que os estudos sociais frequentemente negligenciam a influência dos contextos políticos e culturais amplos, defendendo que o tempo e o espaço são elementos que se impõem para um entendimento pleno do contexto. Entretanto, como pudemos identificar em análises anteriores de estudos organizacionais baseados na prática, mesmo os influenciados pela teoria estruturalista frequentemente encerram sua análise e interpretação no nível - organizacional - meso. Esta tendência é ilustrada por numerosos 
trabalhos empíricos (por ex., Feldman, 2004; Kouamé \& Langley, 2018). Inversamente, as abordagens discursivas, especialmente as caracterizadas pelo pensamento crítico, prestam atenção em contextos particulares, mas dirigem seu interesse principal aos domínios sociais e políticos amplos que ajudam a explicar a produção do local (Grant et al., 2004).

Neste artigo, exploramos uma complementaridade para aproximar as abordagens discursivas e os estudos estruturalistas: resgatar a importância de não limitar a dualidade agência/estrutura a dizeres e fazeres localizados (individuais) ou padrões emergentes como rotinas, discursos, roteiros e gêneros (organizacionais). Em vez disso, ao expandir a noção de contexto e ancorá-la no tempo e no espaço, os estudiosos da prática estarão melhor posicionados para reconectar fazeres e dizeres locais situados a contextos mais macro - políticos, sociais e culturais amplos -, lançando luz, por sua vez, sobre as condições necessárias à reprodução ou transformação das práticas organizacionais ao longo do tempo. Refinando esta ideia, o valor de realizar a conexão entre a análise crítica do discurso e a teoria estruturalista reside em desvelar as práticas organizacionais que se desdobram ao longo do tempo, por meio de um melhor entendimento de como discursos situados no tempo e espaço influenciam e são influenciados pelas estruturas sociais. A literatura já apontou o desafio empírico de ir além da observação da localização temporal das ações comunicativas dentro do contexto de uma conversa, evento ou texto, e a necessidade de tentar aproximar esses elementos com traços institucionais (Heracleous, 2013). Argumentamos que, com esta perspectiva dual, somos capazes de avaliar como as relações de poder se prolongam, reproduzem-se e sofrem resistência por meio de discursos acionados em textos produzidos em contextos cotidianos quando encorados no tempo e no espaço. Além disso, misturar a dualidade de agência e estrutura com o discurso crítico dentro e uma estrutura acionável nos permite entender melhor outra interação importante, mas frequentemente negligenciada: textos e ações, mais precisamente, textos-em-ação. Ao reconhecer e analisar a gama de textos como eles estão sendo produzidos, e ao identificar as práticas discursivas acionadas por esses textos, podemos ampliar nosso entendimento de como as práticas sociais se constituem em ambientes organizacionais e resultam em consequências pretendidas e não pretendidas. Apresentamos a seguir a nossa proposta de aplicação desta lente dual na pesquisa empírica.

\section{APLICANDO O TEMPORAL BRACKETING AOS DISCURSOS: APROXIMAÇÃO, RECUO E VISÃO ATRAVÉS DO TEMPO}

Ao propor uma abordagem analítica que mistura a visão estruturalista e a análise crítica do discurso, combinamos a estratégia de temporal bracketing, conforme a definição de Langley (1999), e as técnicas analíticas críticas do discurso, conforme proposto por Fairclough (1995) - ver a Figura 1.

Aplicar o temporal bracketing aos dados não é uma estratégia metodológica nova em estudos organizacionais (Barley, 1986; Howard-Grenville, 2005). O temporal bracketing tradicionalmente consiste em decompor os dados em períodos sucessivos para examinar como as ações em um período levam a mudanças na estrutura social que afetarão a ação em períodos subsequentes. Esta ferramenta tem sido reconhecida como um exemplo clássico - embora não exclusivo - de uma perspectiva que acolhe uma lógica processual e recursiva intrínseca à dualidade agência/estrutura. Como a interação entre estrutura e ação é difícil de capturar, é mais fácil analisá-las sequencialmente, dentro de brackets sucessivos (Langley, 1999). Um exemplo clássico de temporal bracketing em estudos organizacionais é o modelo sequencial de estruturação de Barley (1986), no qual a noção de rotei- 
ros - contornos de padrões recorrentes de interações que ajudam a definir os papéis dos atores - serve como ligação entre os domínios institucional e da ação.

\section{Figura 1. Temporal bracketing e o quadro conceitual de três dimensões de Fairclough}

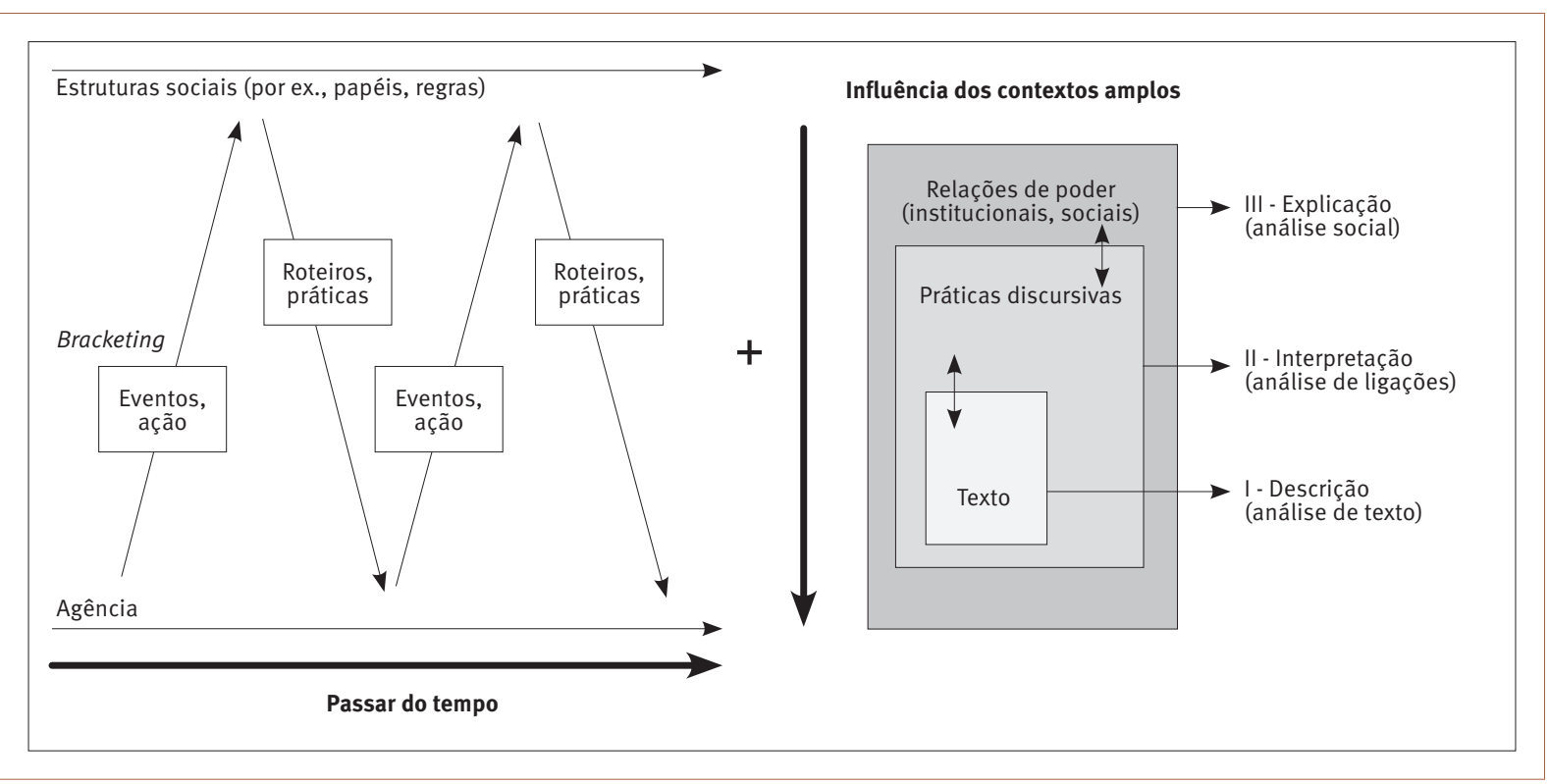

A segunda técnica de pesquisa baseia-se na obra de Fairclough (1995). Nossa escolha foi motivada pela sinergia de dois elementos: a visão de que o quadro conceitual de Fairclough está entre os mais adotados por estudiosos organizacionais e de gestão (por ex., Grant et al., 2001, 2004; Phillips \& Hardy, 2002; Titscher et al., 2000; Wood \& Kroger, 2000; Rodríguez \& Pozzebon, 2010) e a identificação de uma estratégia metodológica acionável de passo-a-passo baseada em sua obra (por ex., Thompson, 2004). 0 quadro conceitual de Fairclough sugere três fases de análise: descrição, interpretação e explicação. Nossa abordagem metodológica - temporal bracketing de discursos - enriquece esses três elementos a partir do quadro desenvolvido por Fairclough, precisamente na medida em que enfatiza a natureza temporal dos eventos organizacionais estudados, incluindo um passo intermediário e imbricado: o bracketing (Figura 2). Aqui, representamos os ciclos recursivos de textosem-ação e práticas discursivas que reforçam certos padrões de ação.

A Fase I consiste na descrição de cada pedaço ou fragmento de texto. Neste processo de análise de texto, os materiais falados e escritos produzidos e consumidos pelos atores são descritos e contextualizados. 0 pesquisador coleta textos-em-ação, o que significa que ele acompanha o momento em que os textos são produzidos pelos atores, estando imerso no contexto da produção e consumo do texto. Na Fase II, enfatizam-se os padrões de identificação e interpretação na produção e consumo dos textos, as chamadas práticas discursivas. Esta fase pode envolver uma variedade de táticas, conforme exemplificado em Cassell e Bishop (2018), incluindo o uso de metáforas (a forma como as pessoas expressam sua vivência e compreensão de uma coisa em termos de outra), modelos (definindo e organizando temas), estórias (sequência de ações e experiências construídas em torno de tramas e personagens ressonantes), representações (o fato de expressar ou denotar por meio de símbolos), imagens (uma descrição falada ou escrita) e conceitos emprestados de outros pontos de vista teóricos ou emergentes da interpretação do pesquisador. Em particular, utilizou-se a metáfora neste trabalho como um 
dos dispositivos interpretativos que os pesquisadores podem mobilizar (ver Cornelissen, Oswick, Christensen, \& Phillips, 2008; Heracleous \& Jacobs, 2008 para mais informações).

Figura 2. O temporal bracketing de discursos

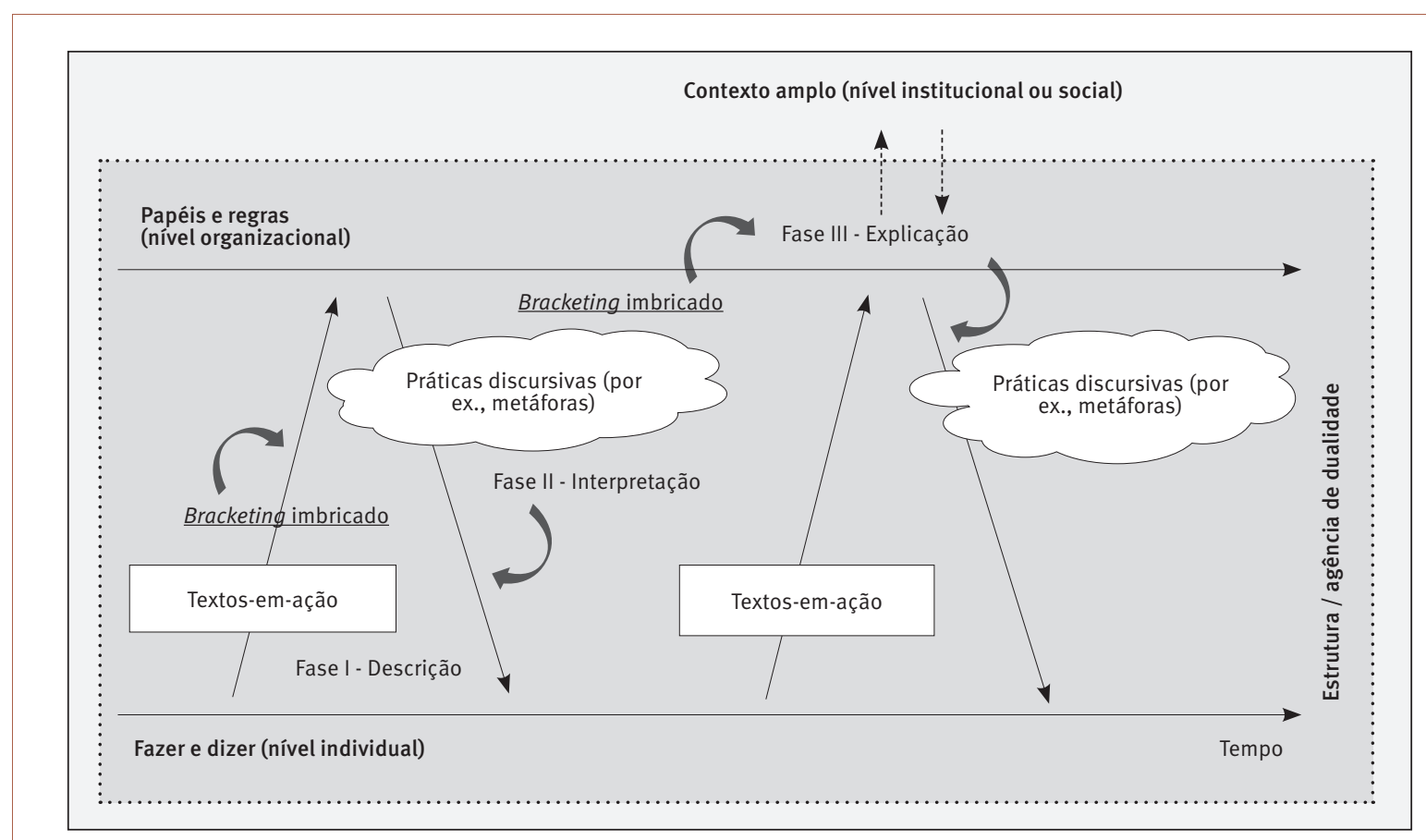

Os dados que emergem das Fases I e II são segmentados em brackets para examinar como os textos-em- $a$ ção contribuem cumulativamente para a estruturação de papéis e regras organizacionais ao reforçar ou transformar estruturas organizacionais existentes ou criar novas estruturas. Na Fase III, o pesquisador tenta desenvolver uma explicação gerada pela conexão das interpretações que se desdobram ao longo do tempo e espaço, isto é, as ligações entre observações prolongadas de interações contínuas, e a análise dos discursos dentro do contexto geral. 0 pesquisador contextualiza as condições e relações de poder que possibilitam e restringem a produção e interpretação dos textos. Nesse sentido, a análise deve levar em conta não apenas a emergência da prática discursiva, mas as observações dos pesquisadores ao longo do tempo (contexto próximo) e sua consideração de contextos amplos (por ex., organizacionais, institucionais, regionais). É importante notar que as etapas do bracketing são imbricadas entre as Fases I, II e III.

Algumas práticas de trabalho de campo facilitam a aplicação da ferramenta do temporal bracketing dos discursos. A primeira é que a coleta de dados de textos-em-ação é ideal para possibilitar que os pesquisadores se tornem mais conscientes das condições - situacionais, institucionais e sociais - que ajudam a explicar o sentido dos textos produzidos e consumidos. Depender exclusivamente de textos coletados a posteriori (frequentemente for meio de dados de entrevistas ou arquivos), embora valiosos, oferece uma imagem parcial do processo estudado. Tanto os gestos, silêncios e vozes ausentes quanto as contradições entre o fazer e o dizer transmitem muito a respeito dos processos organizacionais não capturados quando dependemos apenas de textos reunidos a partir de entrevistas ou documentos. Assim, apoiamos fortemente o uso de dados oriundos da etnografia - de observação participante e não participante. 0 uso de técnicas etnográficas em pesquisas 
sobre gestão é incentivado por um número crescente de estudiosos (Berthod, Grothe-Hammer, \& Sydow, 2017; Jarzabkowski, Bednarek, \& Lê, 2014), pois os sentidos dos textos são condicionados por seus contextos e pelas percepções dos agentes que produzem e consomem esses textos (Heracleous, 2006).

Em segundo lugar, nossa visão do uso do temporal bracketing está mais próxima da lógica recursiva estruturalista, privilegiando, assim, um tratamento minucioso (fine-grained) do material empírico (Pozzebon \& Pinsonneault, 2005). 0 bracketing minucioso decompõe intencionalmente os eventos em efeitos da ação sobre as estruturas, por um lado, e efeitos das restrições institucionais sobre a ação, por outro, ao longo de um estreito continuum de tempo. A estratégia do temporal bracketing em sua forma minuciosa requer uma espécie de densidade de dados, isto é, o pesquisador precisa ser capaz de se aproximar dos dados empíricos e coletá-los intensivamente ao longo do tempo, a fim de examinar cuidadosamente como ações específicas levam a mudanças contextuais que novamente afetam a ação, e assim por diante.

\section{Uma ilustração empírica: o Projeto CONFIG}

A ilustração empírica relatada neste artigo baseia-se em um estudo de caso longitudinal aprofundado, cujo objetivo foi entender os processos pelos quais uma empresa internacional de consultoria (CONSULT) e seus clientes de uma empresa cliente (FIRMA) propuseram-se a implementar uma tecnologia configurável, em um projeto denominado CONFIG. CONSULT é o nome fictício de uma agência de consultoria canadense que vende e implementa pacotes configuráveis. FIRMA é o nome fictício de uma empresa aeronáutica canadense que decidiu implementar uma das soluções configuráveis oferecidas pela CONSULT. CONFIG é o nome fictício do projeto que envolve a implementação pela CONSULT de uma tecnologia configurável na FIRMA. Tecnologias configuráveis são uma grande tendência no setor de tecnologia, e referem-se àquelas tecnologias altamente parametrizáveis, sendo formadas a partir de uma gama de componentes para satisfazer requisitos muito específicos de uma determinada organização.

Utilizamos o termo "ilustração empírica" porque o estudo de caso original foi relatado em outro lugar, sendo aqui aplicado meramente como ilustração da aplicação da abordagem metodológica. 0 trabalho de campo caracterizou-se como intensivo e quasi-etnográfico (Murtagh, 2007). 0 estudo de caso mobilizou técnicas intensas de trabalho de campo (participação em reuniões e observação local prolongada na organização - FIRMA - que recebia a nova tecnologia), análise documental (incluindo todas as mensagens eletrônicas trocadas entre os consultores e clientes) e 16 entrevistas não estruturadas, cada uma com duração de 1 a 2 horas. Um dos pesquisadores teve a oportunidade de contar com uma mesa dentro da FIRMA, onde podiam ficar durante 0 dia todo, uma vez por semana, por seis meses, o que nos proporcionou a oportunidade de observar diretamente o desenrolar dos eventos ao longo do tempo sem muitas restrições em termos de acesso. Tivemos total acesso para observar reuniões formais, analisar documentos e e-mails trocados relacionados ao projeto, realizar visitas regulares, observar e conduzir entrevistas tanto na empresa cliente quanto na de consultoria. Na CONSULT, a equipe do projeto incluiu nove pessoas, das quais entrevistamos as duas consultoras que interagiram intensivamente com os funcionários da empresa cliente: a diretora de projeto Ella, e a gerente de projeto Liz (todos os nomes aqui utilizados são fictícios). Entrevistamos também o gerente de projeto da empresa cliente, Nils.

Com total valorização do contexto, o foco da investigação foram as interações entre os principais atores envolvidos na negociação de seus papéis quando da implementação da tecnologia configurável ao longo do tempo. Os atores do CONFIG decidiram organizar o processo de implementação da tecnologia em dois períodos consecutivos: o Período 1, envolvendo as conversas e reuniões preliminares; e o Período 2, que incluiu a análise 
e parametrização. Respeitamos esses períodos ao realizar o temporal bracketing de nossos dados, porque eles eram não apenas significativos, mas também apresentavam pontos de virada cruciais nas relações que haviam se estabelecido entre os atores.

Começamos a analisar os dados em paralelo à coleta de dados, utilizando a ferramenta metodológica proposta: as Fases I, II e II com uma etapa imbricada, o bracketing. A Figura 3 ilustra esquematicamente o temporal bracketing de alguns dos textos-em-ação e a emergência progressiva de algumas práticas discursivas de conteúdo autoritativo.

\section{Figura 3. Temporal bracketing de discursos aplicado à ilustração empírica}

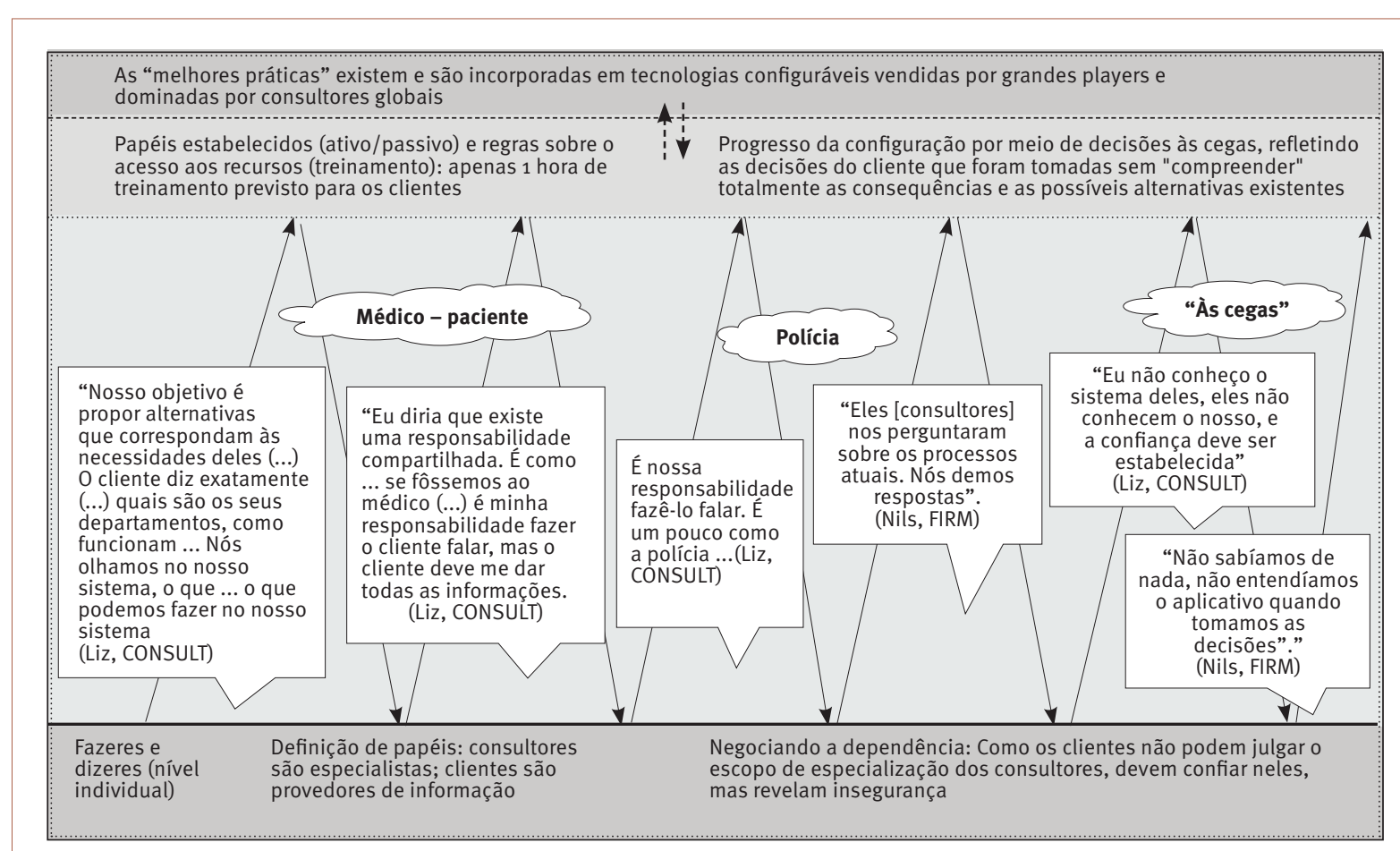

\section{Período 1 - Negociações iniciais entre membros da CONSULT e da FIRMA}

Fase I - Descrição: Uma vez que observamos as negociações iniciais dos papéis e regras do projeto, nossos textos principais nesta fase são notas de trabalho de campo e transcrições ipsis litteris de entrevistas. Desde 0 início, o processo pelo qual os clientes e consultores influenciaram conjuntamente as decisões acerca de como a tecnologia configurável funcionaria foi construído com base em papéis bem definidos e mutuamente acordados, como pudemos observar durante as reuniões formais e conversas informais. Com base em sua expertise técnica, os consultores da CONSULT tinham controle sobre o projeto, e eram formalmente responsáveis por todos os aspectos técnicos da configuração. Os consultores negociaram seu papel como especialistas, enquanto os clientes da FIRMA eram treinados para utilizar, e não para configurar o sistema. Com base em seu conhecimento dos requisitos locais, os clientes forneciam as informações necessárias aos consultores, e faziam escolhas configuracionais à luz de sua compreensão das possibilidades oferecidas pelos consultores. Nesta perspectiva, os clientes assumem o papel de fornecedores de informações, sendo esta divisão de papéis apresentada pelos consultores como algo natural e apropriado. 
Nosso objetivo é propor alternativas que correspondam às necessidades deles (...). 0 cliente nos diz exatamente (...) quais são seus departamentos, como eles funcionam... Nós examinamos o nosso sistema e vemos... o que podemos fazer no nosso sistema (Liz, CONSULT)

A aceitação, e sua expressão verbal, de que os clientes precisam apenas “dizer exatamente quais são os seus departamentos" legitima o papel destes como fornecedores de informações, e propõe uma crença de que as necessidades organizacionais são facilmente transferidas verbalmente. De modo semelhante, aceitar que os consultores "vão examinar seu sistema e ver o que podem fazer" legitima seu papel como especialistas e a crença de que seus métodos efetivamente levam a definições seguras dos requisitos organizacionais e de soluções apropriadas.

Fase II - Interpretação: Práticas discursivas emergiram progressivamente na forma de metáforas, imagens e representações mobilizadas para legitimar esta divisão natural e “apropriada” de papéis. Isto é ilustrado de maneira exemplar na emergência da metáfora "médico-paciente". Observando as trocas entre os principais atores durante o Período 1, compreendemos que uma relação “médico-paciente” estruturava e legitimava a definição de seus papéis, estabelecendo um equilíbrio inicial de poder - quem é o especialista - que influenciou fortemente a negociação que se seguiria. Essas práticas discursivas afetaram diretamente as regras estabelecidas acerca da alocação de recursos: se os clientes são meramente fornecedores de informações, não precisam ser treinados com relação à nova tecnologia, pois os consultores se encarregarão da configuração para eles. Uma vez que não apenas analisamos os textos, mas também observamos os atores no momento da produção desses textos, foi possível observar a fragilidade, a insegurança e um certo processo de “infantilização" nos gestos e respostas do cliente em sua interação com o consultor na primeira reunião importante.

Fase III - Explicação: 0 efeito autoritativo da metáfora médico-paciente ajuda a explicar a construção de um caminho de dependência. Uma evidência clara de que um caminho de dependência estava sendo construído foi a ausência mutuamente acordada de treinamento para os membros da empresa cliente. Na verdade, foi planejado apenas um dia de treinamento antes do lançamento do projeto, uma breve orientação que oferecia uma visão geral e limitada das funcionalidades do pacote. Essas decisões iniciais serviram para estabelecer um cenário com uma série de características que, embora não imutáveis, contribuíam para moldar certas consequências. A falta de treinamento do cliente pode ser entendida como uma consequência direta e lógica de um equilíbrio inicial e mutuamente acordado de poder/expertise, uma consequência inicial do padrão traçado - um caminho de dependência. Limitar o treinamento a um período tão curto - um dia - teria um forte efeito sobre a natureza das intervenções dos clientes e sua participação como membros da equipe do projeto. Aqui, mobilizamos o entendimento de que "caminhos de dependência” entre empresas clientes e consultores devem ser situados no contexto amplo, em nível social ou institucional.

Bracketing imbricado: A descrição e a interpretação foram segmentadas em brackets dentro da explicação e com ela. 0 bracketing recursivo de textos-em-ação e a análise da emergência progressiva de práticas discursivas - no nosso caso, na forma de metáforas e imagens - moldou os textos-em-ação subsequentes. Isto nos ajudou a compreender mais claramente o efeito da temporalidade sobre a estruturação dos papéis e regras ao longo do tempo.

Insights provisórios a partir do temporal bracketing dos discursos do Período 1: A explicação anterior foi estabelecida por dois macroprocessos institucionais independentes fortes e paralelos: a construção da ideia de melhores práticas e as intensas fusões/aquisições/terceirizações que caracterizaram o período desde o ano 
2000 até os dias de hoje. Especialmente no setor de tecnologias da informação e comunicação (TIC), temos testemunhado a emergência e permanência por décadas de um discurso (com "D" maiúsculo) onipresente acerca da existência de "melhores práticas", levando milhares de empresas em todo o mundo a comprar "soluções" de consultorias como o único caminho para consertar problemas informacionais. Este conceito de melhores práticas foi visto como uma ferramenta acionável para facilitar outro fenômeno poderoso: a fusão/aquisição de empresas familiares pequenas e médias por grandes corporações, e a terceirização colateral de inúmeras atividades organizacionais. Quando Ella, Liz e Nils começaram suas “negociações”, a FIRMA havia acabado de ser adquirida por uma empresa maior, e a CONSULT era considerada um dos grandes players em consultoria de TIC, tendo melhores práticas como seu lema.

\section{Período 2 - Atividades configuracionais}

Fase I - Descrição: após a negociação inicial de papéis, foram realizadas atividades principais. Os clientes foram convidados a participar de inúmeras reuniões, chamadas telefônicas e uma intensa troca de e-mails, algumas das quais pudemos observar no local. Em todas essas situações, surgia uma dinâmica comum: os consultores faziam perguntas; os clientes respondiam (fornecedores de informações); com base nas informações fornecidas, os consultores ofereciam diferentes possibilidades de configuração (especialistas); e os clientes selecionavam uma. Por exemplo, durante a segunda reunião importante que observamos, a troca de informações foi conduzida pelos consultores, motivados pela premissa de que, com a ajuda de suas metodologias e agenda de implementações, eles seriam capazes de extrair informações “precisas” sobre os requisitos e especificidades dos usuários. No entanto, para tornar os usuários responsáveis por fornecer informações precisas, a metáfora do "médico" foi novamente mobilizada:

Bem, sim, mas eu diria a você que há, assim, uma responsabilidade compartilhada. É como... se nós fôssemos ao médico, e disséssemos que temos um pouco de dor de cabeça, mas no final, temos uma grande enxaqueca... o médico não vai ser capaz de nos ajudar adequadamente. Na verdade, é... sim, é minha responsabilidade fazer o cliente fala, mas o cliente precisa me dar todas as informações. (Liz, CONSULT)

O processo de configuração baseia-se na desigualdade de poder (os consultores lideram o projeto), uma vez que só os consultores possuem a expertise na ferramenta configurável. Com base em seu conhecimento dos recursos e parâmetros do pacote, e nas informações fornecidas pelos clientes, os consultores "oferecem" diferentes possibilidades de configuração. Os clientes, por sua vez, são dependentes dos consultores: eles não sabem "ler" as possibilidades do pacote, então precisam confiar nas sugestões dos consultores.

Fase II - Interpretação: a desigualdade descrita anteriormente é claramente reforçada por um tipo adicional de metáfora - a da "polícia" - articulada pelos consultores quando descrevem sua relação com os clientes. Durante as duas reuniões formais que observamos, vimos que os clientes tomavam decisões importantes de configuração, mas altamente incertas. Pudemos observar a frequente hesitação dos clientes, bem como suas expressões faciais de falta de compreensão, algo que não poderia ser facilmente capturado em entrevistas. Na segunda reunião formal que observamos, notamos que, em mais de uma ocasião, Liz fez a Nils uma pergunta importante, mas ele pareceu não entender claramente do que Liz estava falando. Eles tentaram explicar seus pontos de vista um ao outro sem sucesso. A certa altura, Nils pareceu envergonhado com a situação, e tomou uma decisão sem entender plenamente as consequências de suas escolhas, e sem ter consciência da adequação destas em relação a outras possíveis escolhas. A observação das interações neste caso realçou a vantagem 
de ir além das entrevistas gravadas, uma vez que pudemos testemunhar um tipo de negociação que caracterizamos como um processo de decisão às cegas.

Não sabíamos nada, não entendemos o aplicativo quando tomamos a decisão. (Nils, FIRMA)

A imagem de estar "às cegas" também foi capturada pela observação de textos-em-ação. Os consultores também trabalhavam com incerteza. Portanto, mobilizavam determinadas estratégias discursivas para legitimar seu papel como especialistas, e, ao mesmo tempo, reduzir sua responsabilidade se as coisas dessem errado. A principal tática descrita é atribuir responsabilidade crescente ao cliente em seu papel como fornecedor de informações, para que a oferta de escolhas ótimas dependa da obtenção das informações certas, sendo esperado que o cliente as forneça.

Se o cliente... não me der as informações... mesmo que eu quisesse dar a ele uma opção mais interessante, eu não poderia, porque eu realmente fui até o fim com a minha pergunta, e ele acabou não trazendo nenhum ponto interessante... então o cliente é responsável pelas informações que ele dá. (Liz, CONSULT)

Fase III - Explicação: o estabelecimento da dinâmica da dependência ao longo do tempo (cujas fundações existiam desde a primeira reunião entre cliente e consultor) somente pode ser entendido se fizermos a conexão com o contexto amplo do setor de TIC e considerarmos a prevalência, característica do século XXI, de terceirizações/fusões. A análise e intepretação desses dados, que parou no nível "meso" - organizacional -, pode encontrar uma explicação para a dependência ser construída no interior da cultura das empresas envolvidas, ou nas personalidades dos atores-chave. Mas uma compreensão do macroclima do setor de TIC e do disseminado predomínio das teorias de melhores práticas provavelmente explica muito a respeito da construção dos caminhos de dependência.

Bracketing imbricado: o relevante aqui é que não poderíamos ter alcançado este grau de entendimento nuançado com base somente em entrevistas. Tampouco tal entendimento poderia ter sido alcançado sem realizar o temporal bracketing das práticas discursivas. A dinâmica de uma pessoa perguntar e a outra hesitar, e finalmente responder sem confiança, não poderia ser facilmente capturada em uma entrevista, principalmente porque os atores ficariam constrangidos em reconhecer sua falta de proficiência e competência nos tópicos em discussão na reunião. A recorrência de tal dinâmica é essencial para a emergência de metáforas "autoritativas", e tal recorrência pode ser capturada de forma viável com a nossa abordagem.

\section{As implicações de teorizar sobre a prática por meio do temporal bracketing dos discursos}

A consequência mais importante da estratégia de segmentação dos discursos em brackets temporais é que ela leva o pesquisador a um profundo entendimento das práticas discursivas por meio de sua investigação das microinterações ao longo do tempo, sem perder o contexto amplo. Primeiro, ao adotar esta abordagem que privilegia a combinação de diferentes técnicas de coleta de dados, estávamos mais bem posicionados para entender o processo de estruturação à medida que ele evoluía. Embora as ações e o discurso - a "manifestação" das estruturas de linguagem e processos linguísticos que trazem as coisas à existência - influenciem fortemente um ao outro, eles não podem ser analisados de forma isolada. Vistos de modo separado, os discursos e ações transmitem relatos parciais da construção da realidade. Dito de outra forma, os atores organizacionais não falam sobre tudo 
o que fazem, não fazem todas as coisas das quais falam, e, às vezes, dizem uma coisa, mas fazem o oposto. Ao focarmos nos textos-em-ação, examinamos os textos conforme eles estavam sendo produzidos e disseminados dentro de uma interação. Em particular, o uso de entrevistas em paralelo a observações prolongadas nos permitiram ir além do conteúdo explícito das entrevistas e levar em conta os elementos tácitos ou não ditos no discurso. Esta abordagem analítica poderia ter focado demais no discurso oficial dos atores envolvidos, perdendo, assim, a oportunidade de capturar como as práticas sociais são estruturadas nas interações.

Em segundo lugar, nossa abordagem facilita a análise da dualidade da estrutura nas práticas discursivas que criam ou mantêm certos padrões de mobilização de recursos e poder de decisão que traduzem os padrões mais amplos de interação social. No caso que ilustramos aqui, utilizando uma abordagem minuciosa do temporal bracketing, fomos capazes de capturar a mobilização prolongada de textos-em-ação ao longo do tempo, destacando a experiência pregressa e conhecimentos acumulados de uma empresa de consultoria por meio do uso de jargão e linguagem técnicos, o que legitimou o papel da consultoria como agente autoritativo. Pudemos observar, por exemplo, os esforços dos consultores em legitimar seus papéis como especialistas em diferentes fases do projeto, quando ocultaram ambiguidades com argumentos racionais sobre metodologias "testadas" e agendas de implementação (que deveriam permitir a elicitação de informações "precisas" sobre o contexto dos usuários) e melhores práticas comprovadas (que deveriam suplantar as "velhas" práticas dos usuários). Se esta análise tivesse adotado uma abordagem transversal, teríamos perdido a oportunidade de ver como a desigualdade de poder foi construída por meio da consolidação dessas práticas discursivas ao longo do tempo.

\section{CONSIDERAÇÕES FINAIS}

Neste artigo, trabalhamos na intersecção da teoria estruturalista e da análise discursiva para propor uma ferramenta metodológica para estudos baseados na prática. Desenvolvemos e descrevemos a aplicação do temporal bracketing de discursos, uma abordagem de teorização que apoia pesquisadores em aproximações, recuos ("zooming in and out”) e visões ao longo do tempo ("over-time") em práticas (Berthod et al., 2017). A abordagem prescreve a observação da produção e consumo de textos-em-ação ao longo do tempo. Por meio do temporal bracketing, segmentamos esses textos em períodos sucessivos, a fim de examinar como eles contribuem cumulativamente para a estruturação das práticas discursivas autoritativas que moldam os fazeres e dizeres subsequentes. A combinação do bracketing com o quadro conceitual proposto por Fairclough relembra os pesquisadores de que a produção e reprodução de práticas sociais depende da conexão de tais práticas entre si e com os contextos político, cultural e social mais amplos.

Nosso artigo contribui para os estudos organizacionais e de gestão de duas maneiras. Primeiro, ao trabalhar “na intersecção", nosso artigo responde a recorrentes chamados para encontrar formas melhores de estabelecer as conexões entre interações localizadas e contextos mais amplos (Nicolini, 2009; Seidl \& Whittington, 2014). Reiteramos o potencial de adotar uma perspectiva dual, na medida em que isto nos oferece a oportunidade de fazer conexões mais nítidas entre textos-em-ação localizados e estruturas sociais mais amplas no tempo. Isto evita que estudiosos baseados na prática restrinjam sua análise ao nível organizacional, e os incentiva a ir além, lançando luz sobre a relação recursiva entre textos-em-ação, práticas organizacionais e estruturas sociais mais amplas.

Em segundo lugar, propomos o temporal bracketing de discursos como uma abordagem de teorização útil para pesquisadores orientados para a prática que estejam comprometidos em se envolver com o campo em tempo real e ao longo do tempo. Esta abordagem valoriza o envolvimento profundo no campo, desde a coleta de dados 
em tempo real e ao longo do tempo até a análise sensível ao contexto, o que é consistente com uma perspectiva prática (Feldman \& Orlikowski, 2011; Jarzabkowski et al., 2007). Um intenso engajamento com o campo ao longo de uma quantidade considerável de tempo pode permitir aos pesquisadores avaliar como os textos-em-ação moldaram e consolidaram progressivamente as práticas discursivas coerentes e conectadas a um contexto amplo, ajudando-os, assim, a entender como tal recursividade representa, na verdade, não apenas a recursividade, mas a vigorosamente buscada noção de dualidade.

Nosso trabalho não é o primeiro a combinar a teoria estruturalista e a análise discursiva, uma vez que outros autores já trabalharam nessas intersecções. Somos provavelmente os primeiros a focar em uma ferramenta metodológica detalhada, que combina, conforme prometido, as conexões local/amplo possibilitadas pelas intersecções. Terminamos propondo uma agenda de pesquisa para estudos futuros, organizados ao longo de três caminhos principais. Primeiro, os estudiosos podem problematizar mais as conexões entre as pontes teóricas e as abordagens metodológicas dedicando-se aos debates existentes sobre teoria e método como interdependentes, e explorando as implicações de novas ferramentas metodológicas de teorização. Em segundo lugar, o papel essencial da temporalidade deve ser revisitado utilizando teorias baseadas na prática. Tem sido apontado que a temporalidade é uma dimensão frequentemente negligenciada em estudos organizacionais, mais genericamente (Leitch \& Palmer, 2010), e nos estudos discursivos em particular (Heracleous, 2013). Certamente acreditamos que um envolvimento mais profundo com a temporalidade será útil. Em terceiro lugar, conforme sugerido por Feldman e Orlikowski (2011), um compromisso com a lente da prática requer um profundo engajamento no campo - observando, coletando e analisado material empírico em tempo real e ao longo do tempo. Isto está em consonância com a crescente valorização das técnicas etnográficas em pesquisa sobre gestão (Berthod et al., 2017; Cunliffe, 2011). Estudos futuros podem avaliar a adequação de outras estratégias de pesquisa na utilização da ferramenta metodológica proposta, especialmente aquelas baseadas na investigação participativa (Reason \& Bradbury, 2008), uma estratégia metodológica poderosa, mas ainda pouco explorada em estudos organizacionais

Trabalhar em 'zonas de transição' é, ao mesmo tempo, arriscado e promissor. Argumentamos que nossa proposta vai um passo além na proposição de uma tática analítica que apoia a 'teorização de estilo conjuntivo'. Como afirmado por Tsoukas (2017), a teorização complexa é propositalmente conjuntiva, e privilegia: “(a) as práticas discursivas e materialmente mediadas nas quais os agentes são lançados, bem como a capacidade intrínseca dos agentes de assumir uma posição por aquilo vivenciam, e de descobrir maneiras possíveis de ser; (b) a singularidade situacional característica de todas as ações práticas; e (c) a natureza de desdobramento da realidade organizacional" (p. 148). Ao propor uma ferramenta metodológica posicionada na intersecção da teoria estruturalista de Giddens e da análise crítica do discurso de Fairclough, contribuímos para os debates contemporâneos em estudos organizacionais.

\section{REFERÊNCIAS}

Balogun, J., Jarzabkowski, P., \& Vaara, E. (2011). Selling, resistance and reconciliation: A critical discursive approach to subsidiary role evolution in MNEs. Journal of International Business Studies, 42(6), 765-786. doi: 10.1057/jibs.2011.13

Barley, S. R. (1986). Technology as an occasion for structuring: Evidence from observation of CT scanners and the social order of radiology departments. Administrative Science Quarterly, 31,78-108. doi:10.2307/2392767
Berthod, O., Grothe-Hammer, M., \& Sydow, J. (2017). Network ethnography: A mixed-method approach for the study of prac tices in interorganizational settings. Organizational Research Methods, 20(2), 299-323. doi:10.1177/1094428116633872

Bourdieu, P. (1990). The logic of practice. Stanford, CA: Stanford university press.

Carter, C., Clegg, S. R., \& Kornberger, M. (2008). Strategy as practice? Strategic Organization, 6(1), 83-99. doi: $10.1177 / 1476127007087154 \mathrm{~V}$ 
Cassell, C., \& Bishop, V. (2019). Qualitative data analysis: Exploring themes, metaphors and stories. European Management Review, 16(1), 195-207. doi: 10.1111/emre.12176

Chouliaraki, L., \& Fairclough, N. (2010). Critical discourse analysis in organizational studies: Towards an integrationist methodology. Journal of Management Studies, 47, 1213-1218. doi: 10.1111/j.1467-6486.2009.00883.x

Cohen, I. J. (1989). Structuration theory: Anthony Giddens and the constitution of social life. New York, USA: St. Martin's Press.

Cornelissen, J.P., Oswick, C., Christensen, L.T., \& Phillips, N. (2008). Metaphor in organizational research: Context, modalities and implications for research - introduction. Organization Studies, 29(1), 7-22. doi: 10.1177/0170840607086634

Cunliffe, A. L. (2011). Crafting qualitative research: Morgan and Smircich 30 years on. Organizational Research Methods, 14(4), 647-673. doi: 10.1177/1094428110373658

Denis, J. L., Langley, A., \& Rouleau, L. (2007). Strategizing in pluralistic contexts: Rethinking theoretical frames. Human Relations, 60(1),179-215. doi: 10.1177/0018726707075288

Fairclough, N. (1992). Discourse and social change. Cambridge, UK: Polity Press.

Fairclough, N. (1995). Critical discourse analysis: The critical study of language. London, UK: Longman.

Fairclough, N., \& Wodak, R. (1997). Critical discourse analysis. In T. A. Van Dijk (Ed.), Discourse studies: A multidisciplinary introduction (Vol. 2, pp. 258-284). London, UK: Sage.

Feldman, M. S. (2004). Resources in emerging structures and processes of change. Organization Science, 15(3), 295-309. doi: 10.1287 /orsc. 1040.0073

Feldman, M. S., \& Orlikowski, W. J. (2011). Theorizing practice and practicing theory. Organization Science, 22(5), 1240-1253. doi: 10.1287 /orsc. 1100.0612

Foucault, M. (1980). Power/knowledge: Selected interviews and other writings, 1972-1977. New York, US: Pantheon Books.

Giddens, A. (1976). New rules of sociological method. New York, USA: Basic Books.

Giddens, A. (1984). The constitution of society. Berkeley, USA: University of California Press.

Giddens, A. (1989). A reply to my critics. In D. Held \& J. B. Thompson (Eds.), Social theory of modern societies: Anthony Giddens and his critics (pp. 249-301). Cambridge, UK: Cambridge University Press.

Giddens, A., \& Pierson, C. (1998). Conversations with Anthony Giddens: Making sense of modernity. Cambridge, UK: Polity Press.

Gioia, D., \& Pitre, E. (1990) Multiparadigm perspectives on theory building. Academy of Management Review, 15(4), 584596. doi: $10.5465 /$ amr.1990.4310758

Grant, D., Hardy, C., Oswick, C., \& Putnam, L. L. (2004). Introduction: Organizational discourse: Exploring the field. In D. Grant, C. Hardy, C. Oswick, \& L. L. Putnam (Eds.), The SAGE handbook of organizational discourse (pp. 1-36). London, UK: Sage Publications.
Grant, D., Keenoy, T., \& Oswick, C. (2001). Organizational discourse: Key contributions and challenges. International Studies of Management and Organization, 31(3), 5-24. doi: $10.1080 / 00208825.2001 .11656818$

Hardy, C. (2001). Researching organizational discourse. International Studies of Management and Organization, 31(3), 25-47.doi: 10.1080/00208825.2001.11656819

Held, D., \& Thompson, J. B. (1989). Social theory of modern societies: Anthony Giddens and his critics. Cambridge, UK: Cambridge University Press.

Heracleous, L. (2006). A tale of three discourses: The dominant, the strategic and the marginalized. Journal of Management Studies, 43(5),1059-1087. doi: 10.1111/j.14676486.2006.00629.x

Heracleous, L. (2013). The employment of structuration theory in organizational discourse: Exploring methodological challenges. Management Communication Quarterly, 20(10),18. doi: $10.1177 / 0893318913504498$

Heracleous, L., \& Barrett, M. (2001). Organizational change as discourse: Communicative actions and deep structures in the context of information technology. Academy of Management Journal, 44, 755 778. doi: 10.5465/3069414

Heracleous, L., \& Jacobs, C. D. (2008). Understanding organizations through embodied metaphors. Organization Studies, 29(1), 45-78. doi: 10.1177/0170840607086637

Howard-Grenville, J. A. (2005). The persistence of flexible organizational routines: The role of agency and organizational context. Organization Science, 16(6), 618-636. doi: 10.1287/ orsc. 1050.0150

Jarzabkowski, (2004). Strategy as practice: Recursiveness, adaptation, and practices-in-use. Organization Studies, 25, 529-560. doi: 10.1177/0170840604040675

Jarzabkowski, P. (2008). Shaping strategy as a structuration process. Academy of Management Journal, 51, 621-650. doi: 10.5465/amr.2008.33664922

Jarzabkowski, P., Balogun, J., \& Seidl, D. (2007). Strategizing: The challenges of a practice perspective. Human Relations, $60(1)$, 5-27. doi: 10.1177/0018726707075703

Jarzabkowski, P., Bednarek, R., \& Lê, J. K. (2014). Producing persuasive findings: Demystifying ethnographic textwork in strategy and organization research. Strategic Organization, 12(4), 274-287. doi: 10.1177/1476127014554575

Kouamé, S., \& Langley, A. (2018). Relating microprocesses to macro-outcomes in qualitative strategy process and practice research. Strategic Management Journal, 39(3), 559-581. doi: $10.1002 / \mathrm{smj} .2726$

Kuhn, T. R., \& Putnam, L. L. (2014). Discourse and communication. In P. S. Adler, P. Du Gay, G. Morgan, \& M. Reed (Eds.), Oxford handbook of sociology, social theory and organization studies: Contemporary Currents (pp. 414-430). Oxford, UK: Oxford Handbooks.

Lacerda, D. S., \& Brulon, V. (2013). Política das UPPs e espaços organizacionais precários: Uma análise de discurso. RAERevista de Administração de Empresas, 53(2), 130-141. doi: 10.1590/S0034-75902013000200002 
Langley, A. (1999). Strategies for theorizing from process data. Academy of Management Review, 24, 691-710. doi: 10.5465/ amr.1999.2553248

Lavarda, R. A. B., Canet-Giner, M., \& Peris-Bonet, F. (2010). How middle managers contribute to strategy formation process: Connection of strategy processes and strategy. RAE-Revista de Administração de Empresas, 50(4), 358-370. doi:10.1590/ So034-75902010000400002

Leitch, S., \& Palmer, I. (2010). Analysing texts in context: Current practices and new protocols for critical discourse analysis in organization studies. Journal of Management Studies, 47, 1194-1212. doi: 10.1111/j.1467-6486.2009.00884.x

Murtagh, L. (2007). Implementing a critically quasi-ethnographic approach. The Qualitative Report, 12(2), 193-215

Nicolini, D. (2009). Zooming in and out: Studying practices by switching theoretical lenses and trailing connections. Organization Studies, 30(12), 1391-1418. doi: 10.1177/0170840609349875

Novak, L., Brooks, J., Gadd, C., Anders, S., \& Lorenzi, N. (2012). Mediating the intersections of organizational routines during the introduction of a health IT system. European Journal of Information Systems, 21(5), 552-569. doi: 10.1057/ejis.2012.2

Orlikowski, W. J. (2000). Using technology and constituting structures: A practice lens for studying technology in organizations. Organization Science, 11, 404-428. doi: 10.1287/ orsc.11.4.404.14600

Phillips, N., \& Hary, C. (2002). Discourse analysis: Investigating processes of social construction. Cambridge, UK: Sage Publications.

Phillips, N., \& Oswick, C. (2012). Organizational discourse: Do mains, debates, and directions. The Academy of Management Annals, 6(1),435-481. doi: 10.5465/19416520.2012.681558

Pozzebon, M. (2004). The influence of a structurationist view on strategic management research. Journal of Management Studies, 41(2), 247-272. doi: j.1467-6486.2004-00431.x

Pozzebon, M., \& Pinsonneault, A. (2005). Challenges in conducting empirical work using structuration theory: Learning from IT research. Organization studies, 26(9), 13531376. doi: $10.1177 / 0170840605054621$

Reason, P., \& Bradbury, H. (2008). The Sage handbook of action research: Participative inquiry and practice. London, UK: Sage Publications
Rodríguez, C., \& Pozzebon, M. (2010). The implementation evaluation of primary care groups of practice: a focus on organizational identity. BMC Family Practice, 11(1), 1-10. doi: 10.1186/1471-2296-11-15

Schatzki, T. R. (1996). Social practices: A Wittgensteinian approach to human activity and the social. Cambridge, UK: Cambridge University Press.

Seidl, D., \& Whittington, R. (2014). Enlarging the strategyas-practice research agenda: Towards taller and flatter ontologies. Organization Studies, 35(10), 1407-1421. doi: 10.1177/0170840614541886

Spee, A. P., \& Jarzabkowski, P. (2011). Strategic planning as communicative process. Organization Studies, 32(9), 12171245. doi: $10.1177 / 0170840611411387$

Taylor, J. R., Cooren, F., Giroux, N., \& Robichaud, D. (1996). The communicational basis of organization: Between the conversation and the text. Communication Theory, 6(1), 1-39. doi: 10.1111/j.1468-2885.1996.tboo118.x

Thompson, M. (2004). ICT, power, and developmental discourse: A critical analysis. The Electronic Journal on Information Systems in Developing Countries, 20(4), 1-25. doi: 10.1007/978-0-387-35634-1_17

Titscher, S., Meyer, M., Wodak, R., \& Vetter, E. (2000). Methods of text and discourse analysis. London, UK: Sage Publications

Tsoukas, H. (2017). Don't simplify, complexify: From disjunctive to conjunctive theorizing in organization and management studies. Journal of Management Studies, 54(2), 132-153. doi: 10.1111/joms.12219

Whittington, R. (2006). Completing the practice turn in strategy research. Organization Studies, 27, 613-634. doi: 10.1177/0170840606064101

Whittington, R. (2010). Giddens, structuration theory and strategy as practice. In D. Golsorkhi, L. Rouleau, D. Seidl, \& E. Vaara (Eds.), Cambridge handbook of strategy as practice (pp. 109-126). Cambridge, UK: Cambridge University Press

Wood, L. A., \& Kroger, R. O. (2000). Doing discourse analysis: Methods for studying action in talk and text. London, UK: Sage Publications

\section{CONTRIBUIÇÕES DAS AUTORAS}

As autoras declaram que participaram de todos os estágios do desenvolvimento do manuscrito. A primeira versão foi desenvolvida por Marlei Pozzebon e Charo Rodriguez. Natalia Aguilar Delgado trabalhou intensivamente nas versões mais recentes. Todas as autoras trabalharam na conceitualização e na abordagem teóricometodológica. As três autoras participaram da revisão teórica. A coleta e análise de dados foi coordenada por Marlei Pozzebon. Todas as autoras trabalharam juntas na redação e revisão final do manuscrito. 